PROCEEDINGS OF THE

AMERICAN MATHEMATICAL SOCIETY

Volume 125, Number 10, October 1997, Pages 3051-3058

S 0002-9939(97)04159-2

\title{
NIELSEN-THURSTON REDUCIBILITY AND RENORMALIZATION
}

\author{
OLIVIER COURCELLE, JEAN-MARC GAMBAUDO, AND CHARLES TRESSER
}

(Communicated by Linda Keen)

\begin{abstract}
Consider an orientation preserving homeomorphism $f$ of the 2disk with an infinite set of nested periodic orbits $\left\{\mathcal{O}_{n}\right\}_{n \geq 1}$, such that, for all $m>1$, the restriction of $f$ to the complement of the first $m$ orbits, from $\mathcal{O}_{1}$ to $\mathcal{O}_{m}$, is $m-1$ times reducible in the sense of Nielsen and Thurston. We define combinatorial renormalization operators for such maps, and study the fixed points of these operators. We also recall the corresponding theory for endomorphisms of the interval, and give elements of comparison of the theories in one and two dimensions.
\end{abstract}

\section{INTRODUCTION}

Thurston's completion [Th] of the work of Nielsen [Ni] on the classification, up to isotopy, of surface homeomorphisms was accomplished at about the same time renormalization group ideas were introduced in the theory of one-dimensional dynamical systems $[\mathrm{Fe}],[\mathrm{CT}],[\mathrm{TC}]$. Some key concepts from these theories, which were formulated quite independently, are quite similar. In this paper we examine some relationships between the two theories. Our aim is to discuss some combinatorial aspects of renormalization group theory for orientation preserving homeomorphisms of the 2-disk, and compare them with what is known in this context for endomorphisms of the interval. In the following discussion, for a sequence $\left(a_{i}\right)_{i \geq 1}$ of integers greater than or equal to 2 , we set

$$
q_{n}=a_{1} \cdot a_{2} \cdots a_{n} .
$$

For $f$ a continuous map of the unit $m$-dimensional disk $\mathbb{D}^{m}$ into itself and $\left(a_{i}\right)_{i \geq 1}$ a sequence as above, we say that $f$ is $\left(a_{i}\right)_{i \geq 1}$-infinitely renormalizable if there exists a sequence of $m$-disks

$$
\mathbb{D}^{m} \supset \mathcal{D}_{1}(f) \supset \mathcal{D}_{2}(f) \supset \cdots \supset \mathcal{D}_{n}(f) \ldots
$$

such that, for each $n$,

$$
f^{j}\left(\mathcal{D}_{n}(f)\right) \cap \mathcal{D}_{n}(f)=\emptyset, \quad \text { for } 0<j \leq q_{n}-1,
$$

and

$$
f^{q_{n}}\left(\mathcal{D}_{n}(f)\right) \subset \mathcal{D}_{n}(f) .
$$

In such a case, by Brouwer's fixed point Theorem, $\mathcal{D}_{n}$ contains one point $x_{n}$ of a periodic orbit $\mathcal{O}_{n}$ with period $q_{n}$. Collections of periodic orbits arising this way have

Received by the editors October 24, 1995.

1991 Mathematics Subject Classification. Primary 58F99. 
well understood special combinatorial structures in the special cases when $f$ is an endomorphism and $m=1$, and when $f$ is an orientation preserving homeomorphism and $m=2$. The map which associates $\left.f^{q_{n}}\right|_{\mathcal{D}_{n}(f)}$ to $f$ is called a renormalization operator and plays an important role in many problems of smooth and holomorphic dynamics. We shall define renormalization operators in the combinatorial context for the cases of dimensions 1 and 2, and compare their dynamical properties. In particular, we shall see that fixed points of combinatorial renormalization operators, as defined in $\S 3$, are much more abundant for endomorphisms in one dimension than for homeomorphisms in two dimensions, despite the fact that permutation groups are finite while braid groups are not.

\section{Combinatorics}

There are classical ways to associate combinatorial objects to periodic orbits of endomorphisms of the interval $I$ (or $f \in \operatorname{End}(I)$ ) and orientation preserving homeomorphisms of the 2-disk $\mathbb{D}^{2}$ (or $f \in \mathrm{Homeo}^{+}\left(\mathbb{D}^{2}\right)$ ).

If $x_{1}<x_{2}<\cdots<x_{p}$ are the $p$ points of a periodic orbit $\mathcal{O}$ of $f \in \operatorname{End}(I)$, let $h$ be the bijection sending $x_{i}$ to $i$. There is a single permutation $\pi$, in fact a cycle, in the permutation group $\mathbf{S}_{p}$ on $p$ elements such that $\left.f\right|_{\mathcal{O}}=h^{-1} \circ \pi \circ h$. We say that $\pi$ represents the action of $f$ on $\mathcal{O}$, and that $f$ realizes the cycle $\pi$. More general permutations can be used similarly to represent the dynamics of $f$ on finite collections of periodic orbits.

Assume now that the $p$ points $x_{1}, x_{2}, \ldots, x_{p}$ of a periodic orbit $\mathcal{O}$ of $f \in$ Homeo ${ }^{+}\left(\mathbb{D}^{2}\right)$ are in the interior of $\mathbb{D}^{2}$, and that $y_{1}, y_{2}, \ldots, y_{p}$ are the $p$ points of a periodic orbit $\mathcal{O}^{\prime}$ of $g \in$ Homeo $^{+}\left(\mathbb{D}^{2}\right)$. We say $(\mathcal{O}, f)$ and $\left(\mathcal{O}^{\prime}, g\right)$ have the same braid type if there exists $h \in$ Homeo $^{+}\left(\mathbb{D}^{2}\right)$ sending $\mathcal{O}$ onto $\mathcal{O}^{\prime}$, such that $h^{-1} \circ g \circ h$ is isotopic to $f$ relative to $\mathcal{O}$. It is straightforward to check that "to have the same braid type" is an equivalence relation. The corresponding equivalence classes are called braid types: if $(\mathcal{O}, f)$ is a representative of the braid type $\beta$, we also say that $\beta$ represents the action of $f$ on $\mathbb{D}^{2} \backslash \mathcal{O}$, and that $f$ realizes the braid type $\beta$. More general braid types can be used similarly to represent the dynamics of $f$ on finite collections of periodic orbits. The braid types with $n$ strands form the braid type set $\mathbf{B} \mathbf{T}_{n}$ which is obtained from the Artin braid group $\mathbf{B}_{n}$ by quotienting by the conjugacies and the center: in the case of a single periodic orbit, any representative $b$ in $\mathbf{B}_{n}$ of a braid type $\beta$ in $\mathbf{B} \mathbf{T}_{n}$ is mapped to a cycle by the canonical projection $\tau$ from $\mathbf{B}_{n}$ to $\mathbf{S}_{n}$ : we say $\beta$ is a cycle if $\tau(b)$ is a cycle (a property which does not depend on the choice of $b$ ).

If $b$ and $b^{\prime}$ are two representatives in $\mathbf{B}_{n}$ of $\beta \in \mathbf{B} \mathbf{T}_{n}$, then $b^{m}$ and $b^{\prime m}$ represent the same element of $\mathbf{B} \mathbf{T}_{n}$ for each $m \in \mathbb{Z}$. As a consequence, any $\beta \in \mathbf{B T}_{n}$ generates a group $\mathbf{G}_{\beta}$ by iteration.

Remark 1. The set of powers of a permutation form a cyclic subgroup of $\mathbf{S}_{n}$. On the contrary, assuming $\beta$ is a cycle, $\mathbf{G}_{\beta}$ is a cyclic group if and only if $\beta$ is an elementary braid type in the sense of Nielsen-Thurston, i.e., $\beta$ is the braid type of a periodic orbit of a rigid rotation of the disk. In all other cases when $\beta$ is a cycle, $\mathbf{G}_{\beta}$ is isomorphic to $\mathbb{Z}$.

A sequence $\left(\mathcal{O}_{i}\right)_{i \geq 1}$ of periodic orbits of $f \in \operatorname{End}(I)$, with periods $q_{i}$, is an $\left(a_{i}\right)_{i \geq 1^{-}}$ cascade of periodic orbits if for each $j \geq 1$, there are pairwise disjoint intervals $\bar{I}_{j, k}$ with $0 \leq k \leq q_{j}-1$ so that each $I_{j, k}$ contains one point of $\mathcal{O}_{j}$ and $a_{j+1}$ points of $\mathcal{O}_{j+1}$ which are all mapped to the same $I_{j, l}$. To each cascade of periodic orbits 
of an endomorphism on the interval corresponds a well-defined sequence $\left(\pi_{i}\right)_{i>1}$ of permutations, where $\pi_{i} \in \mathbf{S}_{q_{i}}$ represents the action of $f$ on $\mathcal{O}_{i}$. We denote by $\mathcal{P}$ the set of such sequences of permutations.

A sequence $\left(\mathcal{O}_{i}\right)_{i \geq 1}$ of periodic orbits of $f \in \operatorname{Homeo}^{+}\left(\mathbb{D}^{2}\right)$, with periods $q_{i}$, is an $\left(a_{i}\right)_{i \geq 1}$-cascade of periodic orbits if for each $j \geq 1$, there are pairwise disjoint disks $D_{j, k}$ with $0 \leq k \leq q_{j}-1$ so that each $D_{j, k}$ contains one point of $\mathcal{O}_{j}$ and $a_{j+1}$ points of $\mathcal{O}_{j+1}$, and $\partial D_{j, k}$ is mapped to a curve homotopic to $\partial D_{j, l}$ relative to the points of $\mathcal{O}_{1}, \mathcal{O}_{2}, \ldots, \mathcal{O}_{j+1}$. Let $\beta_{l}$ be the braid type of $\left(\mathcal{O}_{l}, f\right)$ : then we denote by $\beta_{1} \cup \beta_{2} \cup \cdots \cup \beta_{n}$ the braid type of $\left(\mathcal{O}_{1} \cup \mathcal{O}_{2} \cup \cdots \cup \mathcal{O}_{n}, f\right)$.

To each cascade of periodic orbits of an orientation preserving homeomorphism of the 2-disk corresponds a well-defined sequence $\left(\beta_{i}\right)_{i \geq 1}$ of braid types, where $\beta_{i} \in \mathbf{B T}_{q_{i}}$ represents the action of $f$ on $\mathcal{O}_{i}$. We denote by $\mathcal{B}$ the set of such sequences of braid types (for a different approach to cascades, see [GGH]). By Remark 1, elements of $\mathcal{B}$ generate isomorphic copies of $\mathbb{Z}$ by iteration. Notice also that if $f: \mathbb{D}^{2} \rightarrow \mathbb{D}^{2}$ has an $\left(a_{i}\right)_{i \geq 1}$-cascade of periodic orbits, then, for all $m>0$, the restriction of $f$ to the complement of the first $m$ orbits, from $\mathcal{O}_{1}$ to $\mathcal{O}_{m}$, is $m-1$ times reducible in the sense of Nielsen and Thurston [Ni], [Th].

Remark 2. Let $\left(\mathcal{O}_{i}\right)_{i \geq 1}$ be a cascade of a periodic orbit of $f \in \mathrm{Homeo}^{+}\left(\mathbb{D}^{2}\right)$, and let $\left(\beta_{i}\right)_{i \geq 1}$ stand for the corresponding sequence of braid types. The braid type $\beta_{2}$ is determined by $\left.\beta_{2}^{a_{1}}\right|_{D_{2, j}}$. Thus the braid type $\beta_{1} \cup \beta_{2}$ is determined by the choice of a representative $b_{1}$ of $\beta_{1}$ in $\mathbf{B}_{a_{1}},\left.\beta_{2}^{a_{1}}\right|_{D_{2, j}}$, and an integer $k_{2} \in \mathbb{Z}$ which represents an element of the center of $\mathbf{B}_{a_{2}}$. Similarly the braid type $\beta_{1} \cup \beta_{2} \cup \beta_{3}$ is determined by $b_{1},\left.\beta_{2}^{a_{1}}\right|_{D_{2, j}},\left.\beta_{3}^{q_{2}}\right|_{D_{3, l} l}$, and a pair of integers $\left(k_{2}, k_{3}\right) \in \mathbb{Z}$. If we introduce an integer $k_{1}$ which represents an element of the center of $\mathbf{B}_{a_{1}}$ by which we conjugate $b_{1}$ to another representative of $\beta_{1}$ in $\mathbf{B}_{a_{1}},\left(k_{2}, k_{3}\right)$ has to be replaced by $\left(k_{2}+k_{1} a_{2}, k_{3}+k_{1} a_{2} a_{3}\right)$. Otherwise speaking, the meaningful index $k_{i}$ is not an integer, but a residue class modulo $\frac{q_{i}}{a_{1}}$. This allows us to sometimes use the sequence $\left(\left(\beta_{i}^{q_{i-1}}, k_{i}\right)\right)_{i \geq 1}$ with $k_{1}=0$ instead of the sequence $\left(\beta_{i}\right)_{i \geq 1}$ to represent a cascade of periodic orbits.

Clearly, we have

Proposition 1. An endomorphism of the interval or an orientation preserving homeomorphism of the 2-disk which is $\left(a_{i}\right)_{i \geq 1}$-infinitely renormalizable has an $\left(a_{i}\right)_{i \geq 1}$-cascade of periodic orbits.

To facilitate the discussion, it is useful to extend the definition of cascades to include cases when the $\pi_{i}$ 's or the $\beta_{i}$ 's are not necessarily cycles: we just say cascade in the general case, instead of "cascade of periodic orbits". When the $\pi_{i}$ 's or the $\beta_{i}$ 's are cycles for each $i \geq 1$, the collection $\left(\pi_{i}\right)_{i \geq 1}$ or $\left(\beta_{i}\right)_{i \geq 1}$ is called minimal.

\section{Combinatorial Renormalization}

Let $\left(\pi_{i}\right)_{i \geq 1}$ be the collection of permutations which represents the action of $f \in \operatorname{End}(I)$ on an $\left(a_{i}\right)_{i \geq 1}$-cascade of periodic orbits $\left(\mathcal{O}_{i}\right)_{i \geq 1}$. The combinatorial renormalization operators $\mathbf{R}_{1, a_{1}, j}: \mathcal{P} \rightarrow \mathcal{P}, 0 \leq j \leq a_{1}-1$, are defined by

$$
\mathbf{R}_{1, a_{1}, j}:\left(\pi_{i}\right)_{i \geq 1} \mapsto\left(\pi_{i, j}\right)_{i \geq 1}
$$

where $\pi_{i, j}$ is the restriction to the points in $I_{i+1, j}$ of the permutation $\pi_{i+1}^{a_{1}}$. This definition extends readily to more general cascades. 
Let $\left(\beta_{i}\right)_{i>1}$ be the collection of braid types which represents the action of $f \in$ Homeo $^{+}\left(\mathbb{D}^{2}\right)$ on the complement in $\mathbb{D}^{2}$ of an $\left(a_{i}\right)_{i>1}$-cascade of periodic orbits $\left(\mathcal{O}_{i}\right)_{i \geq 1}$. The combinatorial renormalization operators $\mathbf{R}_{2, a_{1}, j}: \mathcal{B} \rightarrow \mathcal{B}, 0 \leq j \leq$ $a_{1}-1$, are defined by

$$
\mathbf{R}_{2, a_{1}, j}:\left(\beta_{i}\right)_{i \geq 1} \mapsto\left(\beta_{i, j}\right)_{i \geq 1}
$$

where $\beta_{i, j}$ is the restriction to the points in $D_{i+1, j}$ of the braid type $\beta_{i+1}^{a_{1}}$. This definition extends readily to more general cascades.

Remark 3. Like the permutation groups $\mathbf{S}_{n}$ for $n>2$, the Artin braid groups $\mathbf{B}_{n}$ for $n>2$ are not commutative. However, for any finite sequence $\left\{b_{1}, b_{2}, \ldots, b_{m}\right\}$ of elements of $\mathbf{B}_{n}$, the products $b_{1} \cdot b_{2} \cdots b_{m}, b_{2} \cdot b_{3} \cdots b_{1}, \ldots, b_{m} \cdot b_{1} \cdots b_{m-1}$ all represent the same element of $\mathbf{B} \mathbf{T}_{n}$. As a consequence, $\beta_{2}^{a_{1}}$ splits into $a_{1}$ identical braid types with $a_{2}$ strands if $\beta_{1}$ is a cycle.

From this remark we easily get the following result:

Proposition 2. For any $a_{1}>1$, there are cascades (such that $\pi_{1}$ is a cycle), whose images under the $\mathbf{R}_{1, a_{1}, j}$ 's for $0 \leq j \leq a_{1}-1$ are all distinct. On the contrary, the $\mathbf{R}_{2, a_{1}, j}$ 's for $0 \leq j \leq a_{1}-1$ are all the same if $\left(\beta_{i}\right)_{i \geq 1}=\left(\beta_{1}^{\prime}\right)_{i \geq 1}^{m}$ for some $m \in \mathbb{Z}$, where $\beta_{1}^{\prime}$ is a cycle.

In the sequel, we will sometimes use the notation $\mathbf{R}_{2, a_{1}}$ to designate any of the $\mathbf{R}_{2, a_{1}, j}$ 's when $\left(\beta_{i}\right)_{i \geq 1}=\left(\beta_{1}^{\prime}\right)_{i \geq 1}^{m}$ for some $m \in \mathbb{Z}$, where $\beta_{1}^{\prime}$ is a cycle, as in Proposition 2.

\section{Dynamics of RENORMAlization}

For $k \in\{1,2\}$, the product

$$
\mathbf{R}_{k, a_{n}, j} \circ \cdots \circ \mathbf{R}_{k, a_{2}, j} \circ \mathbf{R}_{k, a_{1}, j}
$$

acts (naturally) only on $\left(b_{i}\right)_{i \geq 1}$-cascades with $b_{l}=a_{l}$ for $l \geq n$, so that the combinatorial renormalization operators with $k \in\{1,2\}$ form a pseudo-semigroup.

Given a family of collections of permutations $\left\{\left(\pi_{\alpha, i}\right)_{i \geq 1}\right\}_{\alpha \in A}$, we say $\left(\pi_{i}\right)_{i \geq 0}$ is in the closure of this family if for each $m>0$, there exists an $\alpha$ such that

$$
\pi_{i}=\pi_{\alpha, i} \text { for } 1 \leq i \leq m \text {. }
$$

A similar definition can also be formulated for braid types.

One can then discuss the dynamics of the pseudo-semigroups of combinatorial renormalization operators. For this dynamics, it is plain that periodic points are necessarily labeled by periodic $a_{i}$ sequences (for $i>0$ ), and that fixed points all correspond to cases when all $a_{i}$ 's with $i \geq 1$ are equal to $a_{1}$. We will compare the structure of the fixed points sets in dimensions 1 and 2 .

Proposition 3. For any $a_{1}>1$, any cyclic permutation $\pi$ on $a_{1}$ elements is the $\pi_{1}$ of a minimal fixed point $\left(\pi_{i}\right)_{i \geq 1}$ of all $\mathbf{R}_{1, a_{1}, j}$ 's, $j \in\left\{0,1, \ldots, a_{1}-1\right\}$. For any $a_{1}>1$, any braid type $\beta$ with $a_{1}$ strands is the $\beta_{1}$ of a fixed point $\left(\beta_{i}\right)_{i \geq 1}$ of $\mathbf{R}_{2, a_{1}}$.

Proof. In the 1-dimensional case, we just set $\pi_{1}=\pi$, and for $i>1$ :

$$
\pi_{i}=\pi_{i}^{\prime \prime} \circ \pi_{i}^{\prime}
$$

where $\pi_{i}^{\prime}(k)=\left(k+a_{1}\right) \bmod a_{1}^{i}$ and where $\pi_{i}^{\prime \prime}$ is the identity on all $k>a_{1}$ and acts like $\pi$ on the first $a_{1}$ elements.

The 2-dimensional proof is then obtained by changing notations and using Proposition 2 . 
Theorem 1. For any $a_{1}>1$ and any $j \in\left\{0,1, \ldots, a_{1}-1\right\}$, any cyclic permutation $\pi$ on $a_{1}$ elements is the $\pi_{1}$ of uncountably many fixed points $\left(\pi_{i}\right)_{i \geq 1}$ of $\mathbf{R}_{1, a_{1}, j}$. For any $a_{1}>1$ and any braid type $\beta$ with $a_{1}$ strands is the $\beta_{1}$ of countably many fixed points $\left(\beta_{i}\right)_{i \geq 1}$ of $\mathbf{R}_{2, a_{1}}$.

Proof. In the 1-dimensional case, we set $\pi_{1}=\pi$. For $i>1$, we choose arbitrarily any set $\sigma_{i, 1}, \sigma_{i, 2}, \ldots, \sigma_{i, a_{1}^{i}-2}$ of elements of $\mathbf{S}_{a_{1}}$, and then $\sigma_{i, a_{1}^{i}-1}$ such that

$$
\sigma_{i, a_{1}^{i}-1} \circ \sigma_{i, a_{1}^{i}-2} \circ \cdots \circ \sigma_{i, 1}=\pi \text {. }
$$

Consider now the tree $X$ imbedded in $\mathbb{R}^{2}$ with vertices at level $m$ at $(m, n)$ where $m \in \mathbb{N}$ and $n \in\left\{1,2, \ldots, m^{n}\right\}$ and edges joining each vertex $(m, n)$ to the $a_{1}$ vertices $\left(m+1, a_{1}(n-1)+k\right)$ with $1 \leq k \leq a_{1}$; we say $m+1$ is the level of these edges. Define an automorphism $A_{1}$ of $X$ by letting $\pi$ act on the first level edges of $X$. Clearly $A_{1}$ induces $\pi$ on the first level vertices (all higher level edges and vertices are moved around accordingly). After letting $A_{1}$ act on $X$, let each $\sigma_{1, j}$ act on the second level edges emanating from the vertex $(1, j)$ of $\pi(X)$. This defines a new automorphism, say $A_{2}$, of $X$ and we define $\pi_{2}$ as the permutation on the second level vertices induced by $A_{2}$. More generally, assuming $\pi_{i}$ and the automorphism $A_{i}$ of $X$ have been defined, we construct $A_{i+1}$ by letting each $\sigma_{i, j}$ act on the $(i+1)^{\text {st }}$ level edges emanating from the vertex $(i, j)$ of $A_{i}(X)$, and define $\pi_{i+1}$ as the permutation induced by $A_{i+1}$ on the $(i+1)^{\text {st }}$ level vertices of $X$. The sequence of permutations corresponding to any cascade can be constructed in a similar way and minimality is equivalent to first impose that $\pi_{1}$ is a cycle and then replace equation $(*)$ by the condition that the left hand side of $(*)$ is a cycle for each $i$. The fixed point property is clearly equivalent to impose $(*)$ for each $i$. Because there are at least two choices of factors in the left hand side of $(*)$ for each $i$, this construction shows that for each cycle $\pi_{1}$, there are uncountably many fixed points of $\mathbf{R}_{1, a_{1}, j}$ starting with $\pi_{1}=\pi$. In the 2 -dimensional case, assume $\beta$ is a cycle and set $\beta_{1}=\beta$ (the non-cyclic case is easily deduced). As a consequence of Remark 2, by the fixed point property under $\mathbf{R}_{2, a_{1}}, \beta_{2}$ is completely determined by $\beta$ and $k_{2}$. The choice of $\beta$ and $k_{2}$ further determines the next $\beta_{j}$ 's. More precisely, with the notations of Remark 2, using the definition of a braid type, we have

$$
\left.\mathbf{R}_{2, a_{1}}\left[\left(\beta_{i}^{q_{i-1}}, k_{i}\right)\right)_{i \geq 1}\right]=\left(\left(\beta_{i}^{q_{i-1}}, k_{i}\right)\right)_{i \geq 2}=\left(\left(\beta_{i}^{q_{i}}, k_{i}-\frac{q_{i-1}}{a_{1}} k_{2}\right)\right)_{i \geq 2} .
$$

Hence fixed points must satisfy the relations:

$$
\beta_{2}^{q_{1}}=\beta_{3}^{q_{2}}=\beta_{4}^{q_{3}}=\cdots=\beta_{1}
$$

and

$$
k_{3}-a_{2} k_{2}=k_{2}, k_{4}-a_{3} a_{2} k_{2}=k_{3}, k_{5}-a_{4} a_{3} a_{2} k_{2}=k_{4}, \ldots .
$$

All fixed points starting with $\beta_{1}=\beta$ are obtained this way, and this construction clearly yields fixed points, hence there are countably many of them.

Proposition 4. If $\left(\pi_{i}\right)_{i \geq 1}$ is a fixed point of $\mathbf{R}_{1, a_{1}, j}$, so are all $\left(\pi_{i}^{m}\right)_{i \geq 1}$ 's for all $m \in \mathbf{Z}$. If $\left(\beta_{i}\right)_{i \geq 1}$ is a fixed point of $\mathbf{R}_{2, a_{1}}$, so is $\left(\beta_{i}^{m}\right)_{i \geq 1}$ for any $m \in \mathbb{Z}$. The collection $\left(\pi_{i}^{m}\right)_{i \geq 1}$ or $\left(\beta_{i}^{m}\right)_{i \geq 1}$ is minimal if and only if $m$ is coprime with $a_{1}$ and $\pi_{1}$ or $\beta_{1}$ is a cycle.

Proof. In the 1-dimensional case, the fixed point property reads

$$
\mathbf{R}_{1, a_{1}, j}\left(\pi_{i}\right)=\left.\pi_{i+1}^{a_{1}}\right|_{I_{i+1, j}}=\pi_{i} \text { for } i \geq 1 .
$$


It follows that

$$
\mathbf{R}_{1, a_{1}, j}\left(\pi_{i}^{m}\right)=\left.\left(\pi_{i+1}^{m}\right)^{a_{1}}\right|_{I_{i+1, j}}=\left.\left(\pi_{i+1}^{a_{1}}\right)^{m}\right|_{I_{i+1, j}}=\pi_{i}^{m} \quad \text { for } i \geq 1 .
$$

The minimality statement follows from the fact that when $m$ is not coprime with $a_{1}, \pi_{1}^{m}$ is not a cycle, while when $m$ is coprime with $a_{1}$, it is also coprime with all $a_{1}^{i}$ 's, so that all $\pi_{i}^{m}$ 's are cycles.

The 2-dimensional proof is then obtained by changing notations.

Theorem 2. For each cycle $\pi$, any $j \in\left\{0,1, \ldots, a_{1}-1\right\}$, and any fixed point $\left(\pi_{i}\right)_{i \geq 1}$ of $\mathbf{R}_{1, a_{1}, j}$, there are uncountably many both of minimal and non-minimal fixed points of $\mathbf{R}_{1, a_{1}, j}$ such that $\pi_{1}=\pi$, in the closure of the set of all powers of $\left(\pi_{i}\right)_{i \geq 1}$.

Proof. We shall restrict to minimal fixed points, the non-minimal case being provable in a similar way. Let $\left(\pi_{i}\right)_{i \geq 1}$ stand for a fixed point of $\mathbf{R}_{1, a_{1}, j}$. Then $\left(\pi_{i}^{m}\right)_{i \geq 1}$ is also a fixed point of $\mathbf{R}_{1, a_{1}, j}$ by Proposition 3. Now, $\pi_{i}^{m}$ is a cycle if and only if $m$ is coprime with $a_{1}^{i}$. We denote by $m_{1}, m_{2}, \ldots, m_{s}$ the integers coprime with $a_{1}$ in $\left\{1,2, \ldots, a_{1}-1\right\}$, then by $m_{n_{1}, n_{2}}$ the integers of the form $n_{2} a_{1}+m_{n_{1}}$ in $\left\{1,2, \ldots, a_{1}^{2}\right\}$, and we define inductively the integers $m_{n_{1}, n_{2}, \ldots, n_{k}, n_{k+1}}=n_{k+1} a_{1}^{k}+m_{n_{1}, n_{2}, \ldots, n_{k}}$ in $\left\{1,2, \ldots, a_{1}^{k+1}\right\}$. It is clear that each $\left(\pi_{i}^{m_{n_{1}, n_{2}}, \ldots, n_{i}}\right)_{i \geq 1}$ is a minimal fixed point $\mathbf{R}_{1, a_{1}, j}$ in the closure of the set of all powers of $\left(\pi_{i}\right)_{i \geq 1}$, and that there are uncountably many distinct such objects.

Another proof of Theorem 2 follows from considering conjugacy of the dynamics described by $\left(\pi_{i}\right)_{i \geq 1}$ to some adding machine: see, e.g., section I.4 in [BORT] and references therein.

As a consequence of Remark 1, it is only in the elementary braid type case that one can hope for a counterpart to Theorem 2 .

The fixed points with $\beta_{1}$ an elementary braid type are completely determined by the couple $\left(\alpha, k_{2}\right)$, where $\alpha=\frac{2 p \pi}{a_{1}} \in[0,2 \pi[$ is the angle of the rigid rotation associated to $\beta_{1}$ and the integer $k_{2}$ is defined in Remark 2 (see also [GSuT]). We then have:

Theorem 3. Let $\left(\beta_{i}\right)_{i \geq 1}$ be a fixed point of $\mathbf{R}_{2, a_{1}}$ with $\beta_{1}$ an elementary braid type. Then the set of powers of $\left(\beta_{i}\right)_{i \geq 1}$ contains all fixed points of $\mathbf{R}_{2, a_{1}}$ whose first braid type $\beta_{1}^{\prime}$ is an elementary braid type with $a_{1}$ strands, if and only if $\alpha=\frac{2 \pi}{a_{1}}$ and $k=1$ or $\alpha=\frac{2\left(a_{1}-1\right) \pi}{a_{1}}$ and $k=a_{1}-2$. The two fixed points $\left(\gamma_{i, a_{1}}\right)_{i \geq 1}$ and $\left(\delta_{i, a_{1}}\right)_{i \geq 1}$ so defined for each $a_{1}>1$ are such that for each $i, \gamma_{i, a_{1}}=\left(\delta_{i, a_{1}}\right)^{-1}$. Thus $\left(\gamma_{i, a_{1}}\right)_{i \geq 1}$ and $\left(\delta_{i, a_{1}}\right)_{i \geq 1}$ define a unique fixed point of $\mathbf{R}_{2, a_{1}}$ up to inverse.

Proof. From the computation in the proof of Theorem 1, for any $\beta_{1}$, the fixed point $\left(\beta_{i}\right)_{i \geq 1}$ is characterized by the pair $\left(\frac{2 p \pi}{a_{1}}, k_{2}\right)$. From the same computation, for the $n^{\text {th }}$ iterate the corresponding pair reads $(u, v)=\left(\left\{\frac{2 n p \pi}{a_{1}}\right\}, n\left(\frac{p}{a}+k_{2}\right)-\left[\frac{n p}{a}\right] a\right)$, and it just remains to adjust $p$ and $k_{2}$ so that when $n$ varies through $\mathbb{Z},\left(\frac{a_{1} u}{2 \pi}, v\right)$ varies through $\left\{1,2, \ldots, a_{1}-1\right) \times \mathbb{Z}$. We leave the details to the reader.

Remark 4 . In the case when $a_{1}=2$, the special fixed point singled out by Theorem 3 is, up to inverse, the cascade used by Bowen and Franks to construct the first example of a $C^{1}$ Kupka-Smale diffeomorphism of the 2-sphere with no sink nor source $[\mathrm{BF}]$, as well as the cascade used by Franks and Young to construct the first example of a $C^{2}$ diffeomorphism with the same properties [FY] (see also [GT]). 
In our discussion, there is no 1-dimensional counterpart for these "nicest" fixed points, as described in the following result, but see also Section I.6 and Theorem II.6.10 in [BORT] where one looks at simultaneous fixed points of $a_{1}$ combinatorial renormalization and skewed renormalization operators.

Theorem 4. For each cycle $\pi$ and any $j \in\left\{0,1, \ldots, a_{1}-1\right\}$, there is no fixed point $\left(\pi_{i}\right)_{i \geq 1}$ of $\mathbf{R}_{1, a_{1}, j}$ such that the closure of the set of its powers contains all fixed points of $\mathbf{R}_{1, a_{1}, j}$ with $\pi_{1}=\pi$.

Proof. From the proof of Theorem 1, the number of distinct permutations one can get as $\pi_{i}$ for a fixed point of $\mathbf{R}_{1, a_{1}, j}$ is $\left(a_{1} !\right)^{a_{1}^{i}-1}$, while, by powers of a given fixed point, one gets at most $a_{1}^{i}$ choices for $\pi_{i}$.

\section{Skewed COMbinatorial RENORMalization}

Let $\left(\pi_{i}\right)_{i \geq 1}$ be the collection of permutations which represents the action of an endomorphism $f: I \rightarrow I$ on an $\left(a_{i}\right)_{i \geq 1}$-cascade of periodic orbits $\left(\mathcal{O}_{i}\right)_{i \geq 1}$. The skewed combinatorial renormalization operators $\mathbf{S R}_{1, a_{1}, j}: \mathcal{P} \rightarrow \mathcal{P}, 0 \leq j \leq a_{1}-1$, are defined by

$$
\mathbf{S R}_{1, a_{1}, j}:\left(\pi_{i}\right)_{i \geq 1} \mapsto\left(\pi_{i, j}\right)_{i \geq 1}
$$

where $\pi_{i, j}$ is obtained, from the restriction to the points in $I_{i+1, j}$ of the permutation $\pi_{i+1}^{a_{1}}$, by conjugacy by $\Delta_{\frac{q_{i+1}}{a_{1}}}$, where $\Delta_{n}(i)=n+1-i$. This definition extends easily to more general cascades.

Let $\left(\beta_{i}\right)_{i \geq 1}$ be the collection of braid types which represents the action of a homeomorphism $f: \mathbb{D}^{2} \rightarrow \mathbb{D}^{2}$ on the complement in $\mathbb{D}^{2}$ of an $\left(a_{i}\right)_{i \geq 1}$-cascade of periodic orbits $\left(\mathcal{O}_{i}\right)_{i \geq 1}$. The skewed combinatorial renormalization operators $\mathbf{S R}_{2, a_{1}, j}: \mathcal{B} \rightarrow \mathcal{B}, 0 \leq j \leq a_{1}-1$, are defined by

$$
\mathbf{S R}_{2, a_{1}, j}:\left(\beta_{i}\right)_{i \geq 1} \mapsto\left(\beta_{i, j}\right)_{i \geq 1}
$$

where $\beta_{i, j}$ is obtained as follows:

- take a homeomorphism $h$ realizing the restriction $\beta_{i, j}$ of the braid type $\beta_{i+1}^{a_{1}}$ to the points $\mathcal{O}^{\prime}$ of $\left(\mathcal{O}_{i}\right)_{i \geq 1} \cap D_{i+1, j}$,

-conjugate $h$ by an orientation reversing homeomorphism $k$ to get a homeomorphism $h^{\prime}$, and set $\mathcal{O}^{\prime \prime}=k\left(\mathcal{O}^{\prime}\right)$,

$-\beta_{i, j}$ is then the braid type of $\left(\mathcal{O}^{\prime \prime}, h^{\prime}\right)$.

If $\beta$ is the braid type of $\left(\mathcal{O}^{\prime}, h\right)$, we denote by $-\beta$ the braid type of $\left(\mathcal{O}^{\prime \prime}, h^{\prime}\right)$. The definition of the skewed combinatorial renormalization operator extends readily to more general cascades.

Propositions 2, 3, and 4, and Theorems 1, 2, and 4 have natural counterparts for skewed combinatorial renormalization. Hence, in particular, the symbols $\mathbf{S R}_{2, a_{1}}$ make sense.

The fixed points of $\mathbf{S R}_{2, a_{1}}$ must be of the form $\left((\beta, 0),\left(-\beta, k_{2}\right),\left(\beta, k_{3}\right), \ldots\right)$. When $\beta_{1}$ is an elementary braid type, the fixed points are again completely determined by the couple $\left(\alpha, k_{2}\right)$, described before Theorem 3 . We then have:

Theorem 5. Let $\left(\beta_{i}\right)_{i \geq 1}$ be a fixed point of $\mathbf{S R}_{2, a_{1}}$ with $\beta_{1}$ an elementary braid type. Then the set of powers of $\left(\beta_{i}\right)_{i \geq 1}$ contains all fixed points of $\mathbf{S R}_{2, a_{1}}$ whose first braid type $\beta_{1}^{\prime}$ is an elementary braid type with $a_{1}$ strands, if and only if $\alpha=\frac{2 \pi}{a_{1}}$ and $k=1$ or $\alpha=\frac{2\left(a_{1}-1\right) \pi}{a_{1}}$ and $k=a_{1}$. The two fixed points $\left(\lambda_{i, a_{1}}\right)_{i \geq 1}$ and $\left(\mu_{i, a_{1}}\right)_{i \geq 1}$ so 
defined for each $a_{1}>1$ are such that for each $i, \lambda_{i, a_{1}}=\left(\mu_{i, a_{1}}\right)^{-1}$. Thus $\left(\lambda_{i, a_{1}}\right)_{i \geq 1}$ and $\left(\mu_{i, a_{1}}\right)_{i \geq 1}$ define a unique fixed point of $\mathbf{S R}_{2, a_{1}}$ up to inverse.

Remark 5. In the case when $a_{1}=2$, the special fixed point singled out by Theorem 3 is, up to inverse, the cascade used in [GStT] to construct the first example of a $C^{\infty}$ Kupka-Smale diffeomorphism of the 2 -sphere with no sink nor source.

\section{REFERENCES}

[BORT] H. Bass, M. V. Otero-Espinar, D. Rockmore, and C. Tresser, Cyclic Renormalization and Automorphism Groups of Rooted Trees, Lecture Notes in Mathematics 1621 (Springer, Berlin, 1996). CMP 96:13

[BF] R. Bowen and J. Franks, The periodic points of maps of the disk and the interval, Topology 15 (1976), 337-342. MR 55:4283

[CT] P. Coullet and C. Tresser, Itérations d'endomorphismes et groupe de renormalisation, J. Phys. C5 (1978), 25-28.

[GGH] J. M. Gambaudo, J. Guaschi, and T. Hall, Period-multiplying cascades for diffeomorphisms of the disk, Math. Proc. Cambridge Philos. Soc. 116 (1994), 359-374. MR 95e: 58129

[GStT] J. M. Gambaudo, S. van Strien, and C. Tresser, There exists a $C^{\infty}$ Kupka-Smale diffeomorphism on $S^{2}$ with neither sinks nor sources, Nonlinearity 2 (1989), 287-304. MR 90b:58154

[GSuT] J. M. Gambaudo, D. Sullivan, and C. Tresser, Infinite cascades of braids and smooth dynamics, Topology 33 (1994), 85-94. MR 95a:58078

[GT] J. M. Gambaudo and C. Tresser, Self-similar constructions in smooth dynamics. Rigidity, smoothness and dimension, Comm. Math. Phys. 150 (1992), 45-58. MR 93j:58084

[Fe] M. J. Feigenbaum, Quantitative universality for a class of non-linear transformations, J. Stat. Phys. 19 (1978), 25-52. MR 58:18601

[FY] J. Franks and L. S. Young, A $C^{2}$ Kupka-Smale diffeomorphism of the disk with no sources or sinks, in Dynamical Systems and Turbulence (Warwick 1980), Lecture Notes in Mathematics 898 (Springer-Verlag, Berlin, 1981). MR 83j:58095

[Ni] J. Nielsen, "Investigations in the topology of closed orientable surfaces I, II, and III," Translation by John Stillwell of: "Untersuchungen zur Topologie der geschlossenen zweiseitingen Flaïchen." In Jakob Nielsen: Collected Mathematical Papers (Birkhäuser, Boston, 1986). MR 88a:01070a; MR 88a:01070b

[Th] W. Thurston, On the geometry and dynamics of diffeomorphisms of surfaces, Bull. Amer. Math. Soc. (N.S.) 19 (1988), 417-431. MR 89k:57023

[TC] C. Tresser and P. Coullet, Itérations d'endomorphismes et groupe de renormalisation, C. R. Acad. Sci. Paris Sér. A 287 (1978), 577-580. MR 80b:58043

Section de Mathématiques, Université de Gent̀ve, CP240, CH1211 Gent̀ve 24, Suisse E-mail address: courcell@divsun.unige.ch

INLN, 1361 route des lucioles, Sophia-Antipolis, 06560 Valbonne, France

E-mail address: jmga@ecu.unice.fr

IBM, P.O. Box 218, Yorktown Heights, New York 10598

E-mail address: tresser@watson.ibm.com 